\title{
A Printed Microstrip Yagi Antenna Array for Millimeter-Wave Multi-Sector Applications
}

\author{
G. DeJean and M. M. Tentzeris \\ Georgia Electronic Design Center, School of ECE, Georgia Institute of \\ Technology, Atlanta, GA 30332-0250, U.S.A. \\ email: gdejean@ece.gatech.edu
}

\section{Introduction}

The printed microstrip Yagi antenna array has been utilized in many applications over the last fifteen years. John Huang introduced the first standard design in 1989 for mobile satellite (MSAT) applications, which required a low-cost, lowprofile antenna that covers a $40^{\circ}$ beamwidth between $20^{\circ}-60^{\circ}$ in the elevation plane [1]. The main advantages of a printed Yagi array are the ease of integration with other RF circuitry and the ability to produce "quasi-endfire" radiation. The conventional printed microstrip Yagi array antenna consists of four patch elements: a reflector patch, a driven element, and two director patches. The elements are placed in a line next to each other in the following order: reflectordriven-director 1-director 2. The distance between the elements has to be close enough to couple the electromagnetic fields from the driven element to the remaining elements. The center-to-center spacing between the elements is a function of the free-space wavelength, $\lambda_{0}$, not the guided wavelength, $\lambda_{\mathrm{g}}$. This is associated with the free-space radiation of the antenna. Much progress in microstrip Yagi design has been done in the years after the standard microstrip Yagi array was proposed, including mechanical beam steering, electrical beam steering, parametric study of Yagi arrays, etc. [2-5]. Despite these advances, most of the designs presented have been useful for applications below $15 \mathrm{GHz}$.

Nowadays, there is an increased interest for ultra-broadband mm-wave applications (wireless video transfer at $60 \mathrm{GHz}$ at data rates in excess of $5 \mathrm{~Gb} / \mathrm{s}$ for ranges up to $10 \mathrm{~m}$, mm-wave ad-hoc sensor networks, point-to-multipoint wideband links) that require sectorized radiation patterns (beamwidths $40^{\circ}-60^{\circ}$ ) and high gain (above $10 \mathrm{dBi}$ ) in order to create quasi-omnidirectional radiation pattern and alleviate propagation loss effects. In this paper, a novel printed microstrip Yagi array antenna is proposed as an effective solution for these applications. This is a simple antenna approach that utilizes square elements and a printed feeding network. A maximum directivity of greater than $12 \mathrm{dBi}$ can be achieved with this current design with a radiation efficiency of around $90 \%$ and a beamwidth around 40 degrees. 


\section{Antenna Structure}

The proposed antenna structure is shown in Fig. 1. It consists of two microstrip Yagi arrays that are fed simultaneously to create a "double-printed" Yagi antenna array. The conventional approach of using two microstrip Yagi arrays aligned parallel to each other caused significant radiation in a direction $180^{\circ}$ from the main beam in the azimuth plane. In this approach, the director 1 elements are offset from the parallel configuration and the two individual arrays are diagonally aligned to limit this radiation. As a result, the two individual microstrip Yagi arrays converge to a common director 2 element that is utilized by both arrays. One array emits maximum radiation at $\varphi \approx 30^{\circ}$, while the other array emits maximum radiation at $\varphi \approx-30^{\circ}$. When these arrays are excited simultaneously, constructive field interference occurs and the maximum radiation lies in the positive $\mathrm{x}$ direction $\left(\varphi \approx 0^{\circ}\right)$ with respect to the azimuth plane. All of the patch elements are square. The substrate used in the design is a $200 \mu \mathrm{m}$ thick sheet of RT/duroid 5880 which has a dielectric constant of 2.2 and a loss tangent of 0.0009 at $10 \mathrm{GHz}$. The operational frequency is $32.5 \mathrm{GHz}$. This frequency was chosen for proof of concept and can be easily extended to the $60 \mathrm{GHz}$ range. The dimensions of the patch elements are as follows: reflectors are $4 \times 4 \mathrm{~mm}$, driven patches are $2.95 \times 2.95 \mathrm{~mm}$, the director 1 elements are $2.81 \times 2.81 \mathrm{~mm}$ and the single director 2 element is $2.81 \times 2.81 \mathrm{~mm}$. The angle, $\beta$, between the individual arrays in the "double-printed" Yagi antenna array is $42^{\circ}$. The spacing between each element is $140 \mu \mathrm{m}$. The feeding structure for this design is printed on the same surface with the elements, due to the fact that sometimes the substrate used in the fabrication is not suitable for processing vias in probe feeding techniques. Additionally, aperture-coupling and proximity coupling feeds often require the use of a multilayer substrate such as low-temperature cofired ceramics (LTCC) or liquid crystal polymer (LCP) which can increase the complexity of the design. By printing the feeding structure on the same surface as the elements, many of these complexities are eliminated. The feed network consists of an equal power split Tjunction where the $50 \Omega$ line splits into two $100 \Omega$ lines. Furthermore, the $100 \Omega$ lines pass through the reflector before feeding the driven elements. Hence, a coaxial-to-waveguide (CPW) mode is excited in each Yagi sub array where the reflector is split into two finite ground planes.

\section{Results}

The design in Fig. 1 was simulated using the 3D TLM-based MicroStripes 6.5 by Flomerics, Ltd. The return loss versus frequency is shown in Fig. 2. There are two resonances at $32.2 \mathrm{GHz}$ and $33.4 \mathrm{GHz}$. The lower resonance occurs from the $\mathrm{TM}_{10}$ mode, while the higher resonance comes from a parasitic mode that resonates due to the capacitive coupling of energy between the individual arrays. This resonance could be suppressed by increasing the distance between the individual arrays, but significant sidelobe radiation could contaminate the far field radiation. The relative impedance bandwidth is $7 \%$. The presence of the two resonances helps to widen the bandwidth of the antenna. The radiation patterns at 
33.1 GHz are shown in Figs. 3 and 4. The co-polarized component of the E-plane elevation pattern exhibits maximum radiation at approximately $35^{\circ}$ with a beamwidth of $40^{\circ}$ between $\theta=20^{\circ}$ and $\theta=60^{\circ}$. The maximum directivity is 12.14 $\mathrm{dBi}$. The cross-polarized component of the E-plane elevation pattern is below -35 $\mathrm{dB}$. The $\mathrm{E}_{\theta}$ component of the azimuth plane radiation pattern at an elevation of $35^{\circ}$ is shown to illustrate a reduction of $13 \mathrm{~dB}$ at $\varphi=180^{\circ}$ with respect to the direction of maximum radiation along the positive $\mathrm{x}$ axis. The azimuthal beamwidth is $\approx 60^{\circ}\left(-30^{\circ}-30^{\circ}\right)$. The simulated design exhibits a radiation efficiency of $91 \%$. A lower maximum directivity is exhibited when the angle $\beta$ between the individual arrays was increased with respect to the current value. In addition, significant sidelobes are present which contaminate the far field radiation. If the angle between the individual arrays is too large, the main beam will shift closer to the broadside direction, thus, destroying the Yagi effect. Measured results will be presented at the conference. An extension to 6 antennas in a concentric configuration for the transmitter would enable the full azimuthial ("quasi-omni") coverage of full-space utilizing 6-sectors of $60^{\circ}\left(360^{\circ} / 6=60^{\circ}\right)$, which equals the beamwidth of each element. A $40^{\circ}$ elevation beamwidth would be sufficient for an almost complete, non-intermittent coverage to avoid line-ofsight loss due to humans or other objects]

\section{Conclusion}

A novel, "double-printed" microstrip Yagi antenna array has been proposed for multi-sector mm-wave applications around $30 \mathrm{GHz}$. The design incorporates a printed feeding structure that simplifies the fabrication process, while maintaining a low cost and a low profile. This design can be extended to incorporate H-plane feeding and possibly circular polarization to further increase the maximum data rate. This design approach can be integrated with 3D modules that consist of embedded passives, filters, and MMICs to realize a wireless system-on-package (SOP) RF front end device for very broadband applications around or above 60 $\mathrm{GHz}$.

\section{References}

[1] J. Huang, "Planar microstrip Yagi array antenna," IEEE APS International Symposium, vol. 2, pp. 894-897, June 1989

[2] A. Densmore, J. Huang, "Microstrip Yagi antenna for mobile satellite service," IEEE APS International Symposium, vol. 2, pp. 616-619, June 1991.

[3] D. Gray, J. Lu, D. Thiel, "Electronically steerable Yagi-Uda microstrip patch antenna array," IEEE Transactions on Antennas and Propagation, Volume: 46 , Issue: 5, pp. 605-608, May 1998

[4] S. Padhi, M. Bialkowski, "Parametric study of a microstrip Yagi antenna," Asia-Pacific Microwave Conference, pp. 715-718, Dec. 2000.

[5] Y. Bin, N. Poh, "Microstrip Yagi array on air substrate," IEEE APS International Symposium, vol. 3, pp. 2488-2491, June 2004. 


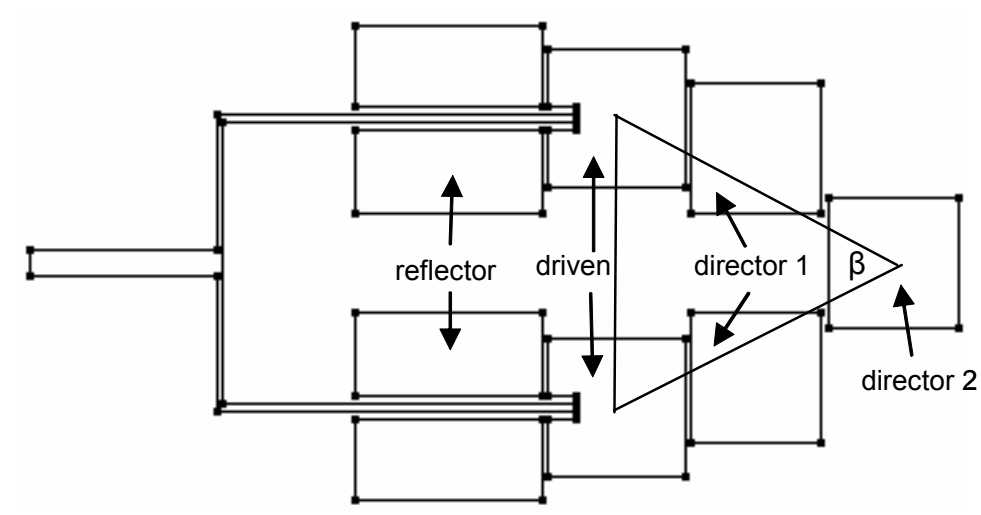

Fig.1 Double-printed Yagi antenna array

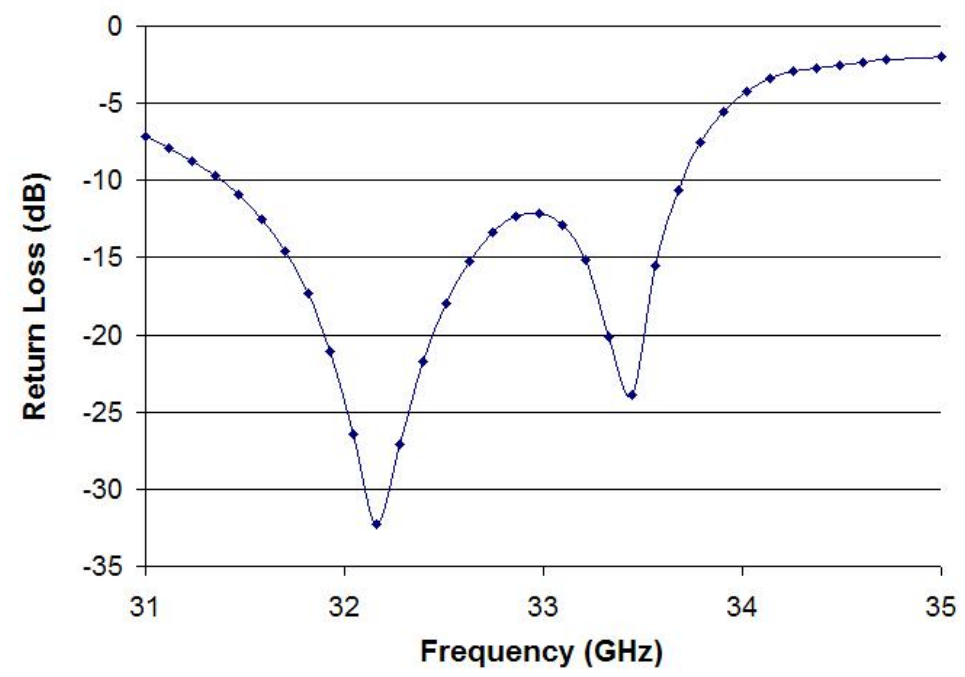

Fig. 2 Simulated return loss versus frequency
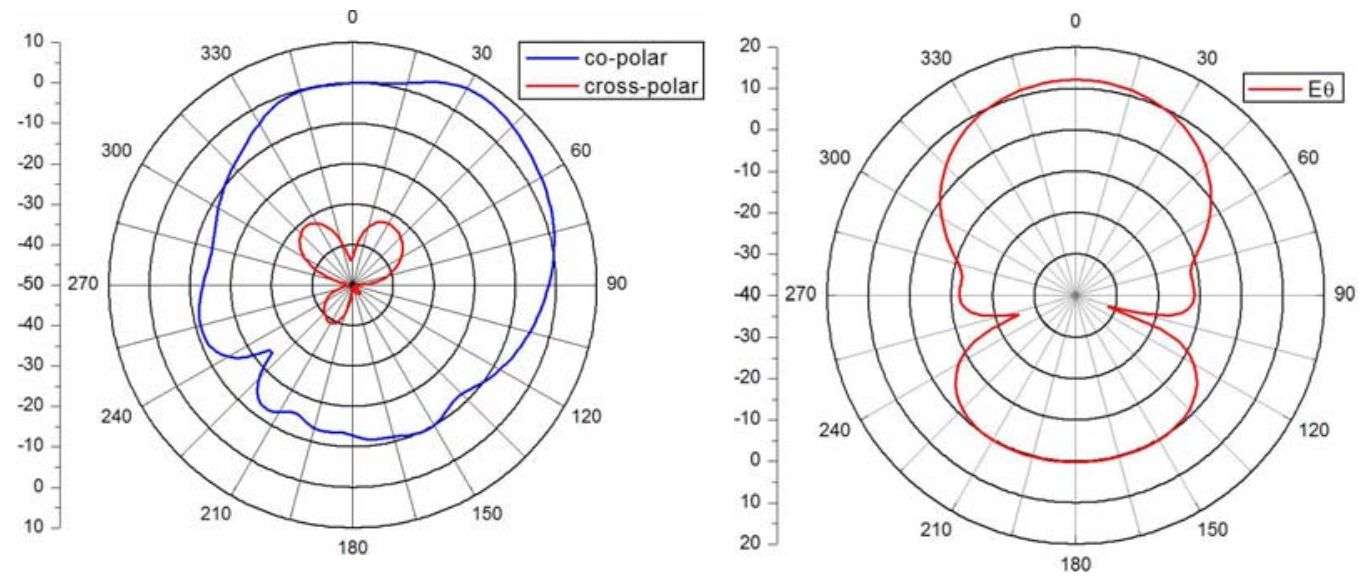

Fig. 3 E-plane co-polar and cross-polar component on elevation plane

Fig. $4 \mathrm{E}_{\theta}$ component on azimuth plane 